\title{
Protocol and Evaluation for Warm-Up Exercise Through Musculoskeletal Simulation
}

\author{
Soichiro Watanabe ${ }^{1,2}$, Hieyong Jeong*3 and Yuko Ohno ${ }^{2}$ \\ ${ }^{1}$ KONOIKE Institute of Technology, Japan \\ ${ }^{2}$ Department of Mathematical Health Science, Japan \\ ${ }^{3}$ Department of Robotics \& Design for Innovative Health Science, Japan
}

*Corresponding author: Hieyong Jeong, Department of Robotics \& Design for Innovative Health Science, Osaka, Japan

\section{ARTICLE INFO}

Received: 㓞 January 24, 2019

Published: 幽 February 11, 2019

Citation: Hieyong Jeong. Protocol and Evaluation for Warm-Up Exercise Through Musculoskeletal Simulation. Biomed J Sci \& Tech Res 14(3)-2019. BJSTR. MS.ID.002542.

\section{ABSTRACT}

It has been reported that many workers at the logistic workplace suffered from musculoskeletal disorders in the lumbar region. Although it has been considered that exercises are one method of effective intervention, there was still no way to prepare exercises. The purpose of the present study was to propose a protocol to prepare the warm-up exercise for logistic workers by using musculoskeletal stimulation, then evaluate the effect of exercise through the clinical test. Three kinds of lifting postures were defined and the each defined posture was stimulated by Open Sim which is the freely available tool for modeling and simulation of movement with the muscle activation. Through the results of the simulation, it was confirmed that 32 types of muscles were activated during three defined postures. According to these results, ten exercising movements were determined with the gymnast instructor. After both qualitative and quantitative evaluation, it was found that the proposed warm-up exercise enabled workers to improve the muscle flexibility as the quantitative evaluation, then the results of qualitative evaluation including the quality of life, visual analysis scale, and so on were connected to the improvement. It was discussed that the proposed protocol to prepare the warm-up exercise was the high feasibility.

\section{Introduction}

In Europe, musculoskeletal disorders (MSDs) are the most common occupation-related diseases, and in the United States, MSDs occupy $5^{\text {th }}$ place in causes of visits to medical facilities. MSDs at the workplace is found not only in specific occupations but also in many types of jobs and kinds of work. Refer to European Survey on Working Conditions (ESWC), the work-related low back disorder is most common MSDs in EU-15 countries [1]. According to the Japanese guidelines for diagnosing and treating low back pain that were issued in 2012, it is believed that there are differences in the ratios of complaints of work related low back disorder depending on the type of occupation, and it has been reported that the figures are $40-50 \%$ for Japanese persons in general, $46-56 \%$ for nurses, and $71-74 \%$, the highest ratio of all, for workers at the transport and logistics workplace. The fact that heavy labor that has a large physical burden is a risk factor for MSDs is a consistent finding in many studies. In addition, it has been pointed out that posture during work is important, and work that involves bending or rotating the trunk of the body, and work wherein the worker cannot change his posture periodically, causes an increased incidence of work related low back disorder [2].

One reason why the complaint rate for work-related low back disorder in the transport and logistics industries is the highest of all industries may well be that in these two industries, work involving the lifting of heavy objects accompanied by bending and rotating of the trunk of the body, is common. Figure 1 An importance of early detection and exercise of musculoskeletal disorders (MSDs) in order to reduce the ratio of occupational MSDs. Figure 1 shows an importance of early detection and exercise for MSDs in order to reduce the ratio of occupational MSDs. In our previous studies $[3,4]$, methods to recognize characteristics of health experts at the logistics workplace were proposed in order to figure out what was the know-how to reduce the burden of the human body during the 
lifting. In this study, we are interested in what are the characteristics of experts with MSDs. Although it is necessary for most workers to take a proper management when they feel the indisposition, it is impossible to determine the rehabilitation at the end by themselves. Because there is no way to express the painful degree of MSDs by themselves through the heavy labor quantitatively. Thus, if characteristics of experts with MSDs are clear, it is possible to determine the optimistic exercise as one kind of rehabilitation for the prevention of severe MSDs by themselves.

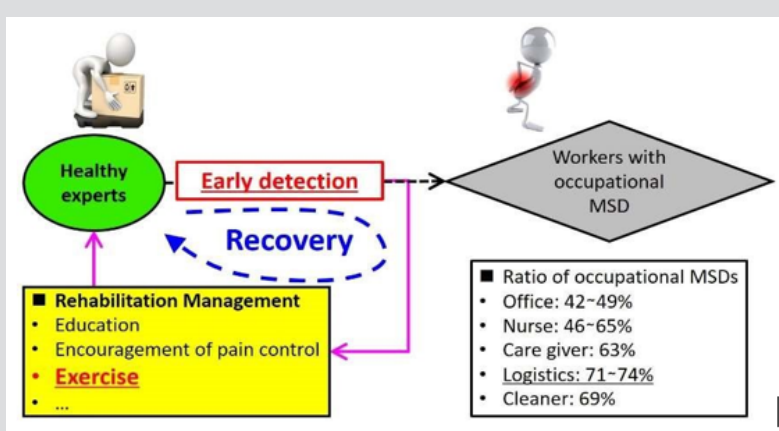

Figure 1: An importance of early detection and exercise of musculoskeletal disorders (MSDs) in order to reduce the ratio of occupational MSDs.

Meanwhile, it has been reported that exercise is one effective innervation of work-related back disorders. In prior studies, various movement methods have been tested. However, according to John (2008), it is believed that in prior studies, information about the protocols of exercise and the technical details of specific exercises is lacking, and it has been noted that exercise is combined in the guidelines and systematic reviews as well, and the importance due to differences of types of exercise has been overlooked [5]. Owing to this, in the European guidelines, it is reported that it is not possible to recommend specific types of exercise that can be selected and performed [6]. Namely, although there have been a lot of case studies to evaluate some exercise, there was no protocol to prepare some necessary exercise. Therefore, the purpose of the present study was to propose the protocol to prepare the warm-up exercise for logistic workers by using musculoskeletal stimulation, then evaluate the effect of exercise through the clinical test.

\section{Human Subjects and Methods}

\section{Human Subjects}

Thirty-four human subjects participated in this study; ten skilled experts of KONOIKE Transport Co., Ltd. [7] without any subjective symptom (Group 1; age: $35.3 \pm 5.9$ years old; height: $1.72 \pm 0.09 \mathrm{~m}$, body mass index (BMI): $24.08 \pm 3.07 \mathrm{~kg} / \mathrm{m}^{2}$ ), ten unskilled novices of university without any subjective symptom (Group 2; age: 29.7 \pm 2.6 years old; height: $1.74 \pm 0.05 \mathrm{~m}$, BMI: $21.02 \pm 4.71 \mathrm{~kg} / \mathrm{m}^{2}$ ), and fourteen experts of KONOIKE Transport Co., Ltd. with MSDs of subjective symptom (Group 3; age: $38.1 \pm 7.2$ years old; height: 1.70 $\pm 0.06 \mathrm{~m}$, BMI: $23.48 \pm 1.75 \mathrm{~kg} / \mathrm{m}^{2}$ ). There was no big difference of BMI between the three groups. No human subject reported a major back or lower limb pathology, use of medication, or a history of neurologic disease that might influence standing balance. During the experiment, all of the human subjects were directed to close their eyes in order to eliminate the influence of visual feedback on postural balance. Their findings are that the cerebellar vermis efferent system is involved in the active maintenance of body balance, and the visual association cortex contributes to its stability possibly by monitoring three-dimensional orientations in space while standing [8].

Written informed consent was obtained from all subjects after a full explanation of the experimental purpose and protocol. Moreover, the experimental protocol in this study was approved by the inquiry committee of study intended for human "Osaka University Hospital Ethics Committee” (no. 15408, 2015).

\section{Muscle Activity by Musculoskeletal Simulation}

Posture when raising heavy objects differs greatly, depending on the individual and the lifting method. Moreover, it is no easy matter to decide which posture in a series of lifting motions is defined as the lifting posture. Accordingly, in order to define the most specific static posture, a pre-set posture, which is the posture that has been originally installed in 3DSSPP, is employed [9]. In this study, three kinds of postures for lifting heavy objects were defined: standing lift, stoop lift, and squat lift, all of which are methods for lifting heavy objects in pre-set posture. In addition, with respect to the active muscle calculation, it is believed that this involves a large number of just the skeletal muscles of the human body. Comprehensively grasping the movements of those muscles by actual measurement is impossible, even if modern scientific techniques are used. Accordingly, the three heavy object lifting postures defined by 3DSSPP are reproduced inside Open Sim [10]. The setting method of posture in the two simulation soft is difference therefore transformational processing is needed as shown in Table 1. In this study, The Full Body Running Model is used as the musculoskeletal model. This model has 29 degrees of freedom in a three-dimensional space and has a total of 92 types of lower limb muscles, 46 types for the right half of the body and 46 types for the left half of the body. By reproducing with OpenSim the three postures defined by 3DSSPP and performing a static analysis, it is possible to compute the action of the muscles during the defined posture.

Table 1: Transformational method between two different simulation software.

\begin{tabular}{|c|c|c|c|}
\hline Parts & 3DSSPP & Processing & Open Sim \\
\hline Arm & Elbow Included & $180^{\circ}$ - 3DSSPP & Elbow flex \\
\hline Arm & $\begin{array}{c}\text { Shoulder } \\
\text { Vertical }\end{array}$ & $\rightarrow$ & Armaflex \\
\hline Upper Leg & Hip Vertical & $\rightarrow$ & hip flexion \\
\hline Lower Leg & Knee Included & 3DSSPP - $180^{\circ}$ & knee angle \\
\hline Lower Leg & Ankle Included & $\begin{array}{c}\text { if }<90^{\circ} \rightarrow 90^{\circ}- \\
3 \mathrm{DSSPP}\end{array}$ & ankle angle \\
\hline
\end{tabular}




\begin{tabular}{|c|c|c|c|}
\hline & & $\begin{array}{c}\text { if } \mathrm{x}>90^{\circ} \rightarrow \\
-3 \mathrm{DSSPP}+90^{\circ}\end{array}$ & ankle angle \\
\hline Torso & $\begin{array}{c}\text { Flexion/ } \\
\text { Extension }\end{array}$ & 3DSSPP $-90^{\circ}$ & $\begin{array}{c}\text { lumbar } \\
\text { extension }\end{array}$ \\
\hline
\end{tabular}

First of all, an examination of the static posture for thoroughly moving the muscles that are activated during the lifting of heavy objects is performed with Open Sim. In the formulation of exercises as well, an analysis of the static posture is performed in order to evaluate the most specific posture. In recent research, it has been reported that when static stretching is performed before sports, this exerts an adverse effect on the muscles, causes performance in sports to decline, and is linked to injuries and muscle damage [11]. Compared to this, it has been confirmed that dynamic stretching has the effect of raising performance if it is performed before sports. According to the guidelines issued by the American College of Sports Medicine, dynamic stretching is defined as a stretching method of moving slowly from a specific posture to a specific posture and continuing to expand the scope and range of motion gradually each time the stretching is repeated [12]. In this study, this specific static stance is depicted during the course of a series of dynamic stretches. It was decided that a static posture would be chosen in order that the target muscles would be activated a minimum of two times. The formulation was done while gymnast instructors were consulted, and verification was conducted in order that difficult postures and complicated postures were not included. In addition to confirming the movability of the target muscles with Open Sim.

\section{Evaluation of Exercise Effects}

\section{Clinical Test}

A clinical trial of the effects of the formulated exercises was conducted, and evaluation of the effects was performed. In addition, qualitative evaluations such as a questionnaire survey and quantitative evaluations such as physical measurements were conducted concerning the improvement of work-related low back disorder and the effects of prevention indirectly. The study subjects were chosen randomly from male employees between the ages of 20 and 50 of KONOIKE TRANSPORT CO., LTD. The workers participated voluntarily based on their own free will. The conditions for participation in the experiment are indicated below:

I. At the time of participation in the investigation, the subject must not have any specific low back pain and not be going to the hospital regularly for treatment due to some disease or another.

II. The subject must have been engaged in the logistics business for at least 3 years and practice, for at least 3 years, the radio calisthenics performed inside KONOIKE TRANSPORT CO., LTD.

A single-arm study was used as the study design. For the schedule, these exercises were practiced only on weekdays, twice per day, at $9 \mathrm{AM}$ and $1 \mathrm{PM}$. In addition, they were performed continuously for 12 weeks. Measurements were made in Week 0 and Week 12. The issue of whether or not the study subjects were performing the exercises was strictly managed with a checklist.

\section{Evaluation}

In order to verify the ameliorative effects on work-related low back disorder as well as the causes of their onset related preventive effects, measurements were conducted for the following three items.

\section{Qualitative Evaluation}

Information about the current pain state of the low back of the study subjects was collected by employing $100 \mathrm{~mm}$ VAS. The length of the scale was evaluated as $0 \mathrm{~mm}$ (least) to $100 \mathrm{~mm}$ (most intense pain). Evaluation items were collected that related to the quality of life (QOL). The Oswestry Disability Index (ODI) was implemented in questionnaire form. It is composed of 10 items that correspond to various activities in daily life and is evaluated with a 6-point scale (0-5). Finally, the score is expressed by a figure of $0-100 \%$, and the larger the percentage is, the higher the degree of disability in the low back is [13]. The Roland-Morris Disability Questionnaire (RDQ) is an evaluation sheet that was designed by Roland and Morris (1983) and translated into Japanese. This describes a "yes" or "no" 24 items of conditions where the study subjects feel difficulties due to a disability of the low back. The larger the number of marked questions is, the higher the extent of the disability in the low back is [14]. The QOL SF-36 v2 was implemented in questionnaire form and is evaluated by the component summary score. Here the component summary scores are composed of three things: the component summary score that expresses physical health (PCS: Physical component summary), that expresses mental health (MCS: Mental component score), and that expresses the role/social health (RCS: Role-social component score). It is recommended evaluating by an appropriate combination of the comprehensive scale and specific scale. The comprehensive scale expresses the overall state of health, and QOL SF-36 v2 corresponds to this. Compared to this, the specific scale was created in order to reflect the specific medical conditions of a disease, and the ODI, as well as the RDQ, correspond to this [2].

\section{Quantitative Evaluation}

Indices related to physical measurements were performed. At first, the finger-floor distance (FFD) was measured. The subject bent the trunk of the body forward and the distance between the fingers that were hanging down wards and the floor was measured. In a standing position with the body bent forward, it is believed that a decline in flexibility is related in a statistically significant manner to low back pain, and the flexibility of the thighs and low back were chiefly measured [15]. Then, Muscle mass (MM) was measured. This was measured with the dual type body composition meter RD907 made by Tanita Co., Ltd. In the MM measurement, it has been reported that low muscular strength is one factor in the onset of 
low back pain, and the changes in lower limb muscular strength were measured [2]. Then, the range of each joint motion (ROM) was measured. Measurements were conducted in conformity with the items enacted by the Japanese Orthopedic Association. There were reports about the ROM of the joints to the effect that the ROM of the hip joint was narrower in persons complaining of the low back pain than it was in persons without any complaint. Measurements for the ROM of the lower limb joints, first among them the ROM of the hip joints, were performed [16].

Finally, the sway direction of the center of pressure (CoP) during lifting by using the Wii Balance Board (WBB) was measured. The CoP orbit when a subject remains motionless for $5 \mathrm{~s}$ while holding a heavy object (18 kg) was measured, and CoP orbits were measured five times. In the measurement of the sway of CoP during heavy object lifting by using WBB, there were reports to the effect that the balance ability of persons complaining of low back pain was inferior to that of healthy persons. In addition, the decline in balance ability was related to the future onset of low back pain [17]. Since the experimental subjects in this study were logistics workers, the sway of CoP when lifting the load, namely holding a heavy object, is applied was measured. Although there are various evaluation indices for the sway of COP, in this study evaluation was performed for the total length (LNG), the mean sway velocity (SV), and the environmental area (EA) of CoP, which are the most representative. In addition, a comparative trial with the Force Place was performed for the WBB, and the WBB was established as the device for evaluating CoP [18].

\section{Measurement of Vertical Ground Reaction Forces}

Figure 2 showed an experimental set-up for measurement and an overview of the experimental environment. The experimental system consisted of three pieces of equipment: a Wii Balance Board (WBB) for measuring CoP parameters, a computer for saving and analyzing data, and a mass of $18 \mathrm{~kg}$. The WBB $(23 \times 43 \mathrm{~cm}$ platform), which was designed to support people weighing up to $136 \mathrm{~kg}$ and was probably more accurate than a typical bathroom scale, fed data into the computer through a Bluetooth connection [14]. The force sensors which consisted of a metal beam and the strain gauge that acted as an uni-axial force transducer have been reported to be linear with the center of pressure (CoP) noise levels of approximately $\pm 0.5 \mathrm{~mm}$ [15]. Although originally designed as a video game controller, the WBB became a proven tool for assessing the vertical ground reaction force (VGRF) and CoP position and was confirmed to be both accurate and reliable. Moreover, the WBB provided a portable and inexpensive balance assessment system that was widely available. Several studies demonstrated the validity and test-retest reliability of the WBB, where the vGRF and CoP position was measured, and the obtained data were compared to those from an identical study conducted using a laboratory-grade force plate. These studies found the WBB to provide reliable and consistently repeatable data. Thus, it was highly probable that an analysis of the different VGRF and CoP position was verified by the WBB in order to confirm the feasibility of field application $[18,19]$.

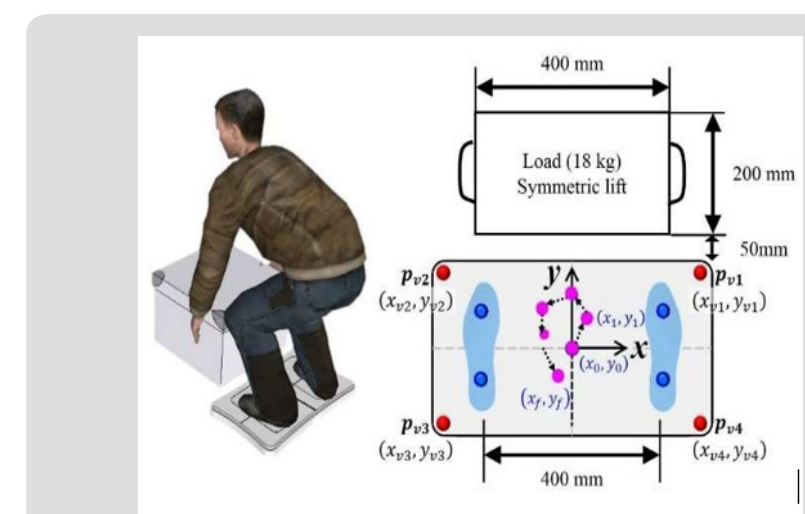

Figure 2: Experimental set-up for measurement and an overview of experimental environment.

Four pressure sensors were installed on the WBB as shown in Figure 2. Time series data of vGRF were measured by the sum of four pressure sensors $(f)$ of $p(t), p(t), p(t)$, and $p(t)$,

$$
f=\frac{\sum p(t)}{f}
$$

where $f$ was the body weight during the quiet standing as the body mass. We could represent $f$ with the ratio of $f$ (body mass) during the lifting. The distributed floor reaction force could be replaced by a single force acted on zero-moment point (ZMP). In general, we considered that the ZMP was equal to the CoP [20]. We could calculate the CoP trajectory when we knew the geometry information of four pressure sensors as shown in Figure 2. All measured data were stored in the computer with a sampling time of $20 \mathrm{~ms}$.

\section{Results}

\section{Results of Vertical Ground Reaction Forces}

Figure 3 showed results of measured vGRF parameters for three different groups during the symmetric lifting posture (Group 1: (a) all of the skilled experts; Group 2: (b) all of the unskilled novices; Group 3: (c) all of the experts with MSDs of subjective symptom). The horizontal and vertical axes represent the ratio of motion cycle such as $T(i=1,2,3)$ and body mass such as $F(i=$ $1,2,3)$, respectively. Three red colored circles and lines indicated the mean \pm standard deviation (SD): $(T, F),(T, F)$, and $(T, F)$ as shown in Figure 3. It was shown that there were no big individual differences between motion cycle and the ratio of body mass for Group 1, but there were big differences for Group 3. It was necessary to confirm the reliability of measured vGRF parameters. Reliability coefficients of knee joint extension function were very high (right leg: 0.94; left leg: 0.95). ICCs for each parameter were high (ICC $=$ $0.70 \sim 0.95)$. Significant differences were found in vGRF and phase at hip-lift off ( $F$ and $F, T$ and $T$ ), the impulse between the beginning of the stand-to-sit (StSi) movement and hip-lift off $(P)$, the impulse 
between hip-lift off and the appearance of peak vGRF $(P)$, and the impulse between the appearance of peak vGRF and the completion of the sit-to-stand (SiSt) movement $(P)$. Their ES were very high
$(\mathrm{ES}=0.57 \sim 1.03)$. From this point of view, reliabilities of vGRF parameters were considered to be good, thus a mean value of three trials was used for further detailed analysis.

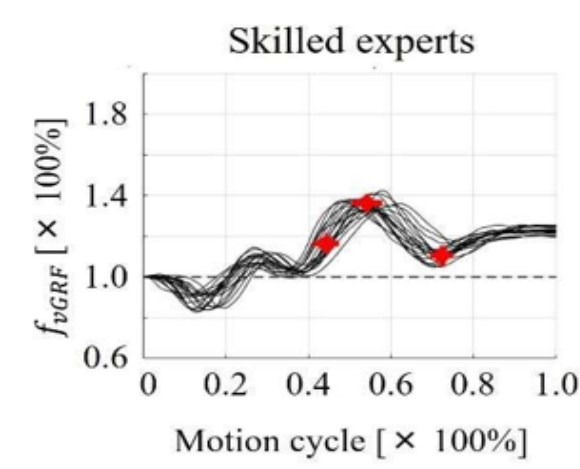

(a) Results of measured vGRFs for all of skilled experts $(n=30)$.

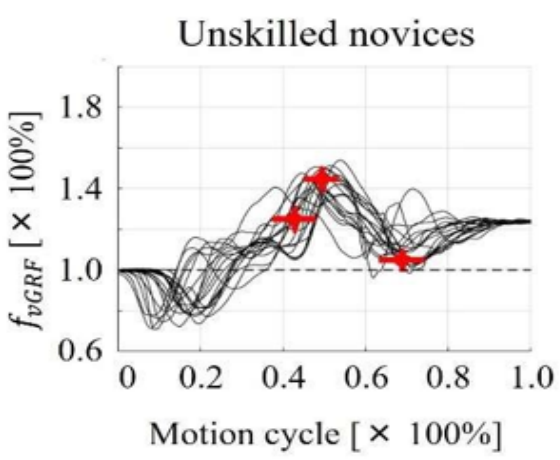

(b) Results of measured vGRFs for all of unskilled novices $(n=30)$.

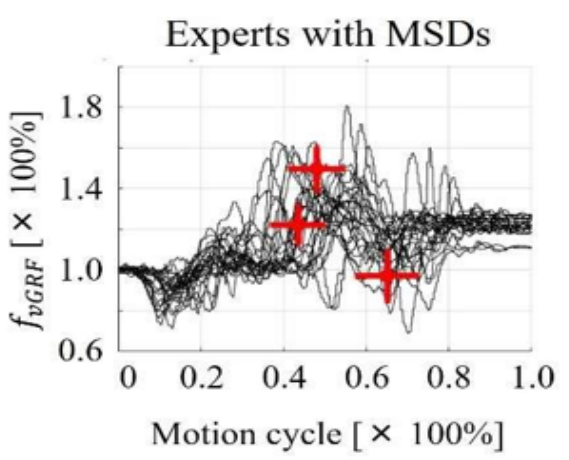

(c) Results of measured vGRFs for all of experts with MSD ( $n=42)$.

Figure 3: Results of time series data of measured vertical ground reaction forces (vGRFs) for all of the groups.

\section{Influence of Musculoskeletal Disorders on Lifting Posture}

It was found that standard deviation (SD) data of $T$ for Group 3 were much shorter $(0.85 \pm 0.46$ times) than those for Group $1(\mathrm{P}<$ $0.001)$. Those for Group 2 were $0.87 \pm 0.37$, and there was no big statistical difference $(\mathrm{P}>0.05)$. Furthermore, it was found that the SD data of $F$ for Group 3 were much larger ( $4.22 \pm 1.42$ times) than those for Group $1(\mathrm{P}<0.001)$. Those for Group 2 were $1.89 \pm 0.63$, and there was a big statistical difference $(\mathrm{P}<0.001)$. Thus, it was considered that characteristic of SD data of shorter $T$ and larger $F$ for Group 3 represented the influence of MSDs on symmetric lifting posture.

\section{Difference of Hip-Lift Phase}

In terms of the difference of hip-lift off phase, it was found that Group 3 were much smaller $(0.59 \pm 0.02$ times) than Group 1 (P $<0.001)$, although there was no big statistical difference of $(T-$ $T)(\mathrm{P}>0.05)$. Those for Group 2 were $0.78 \pm 0.26$, although there was also no big statistical difference of $(T-T)(\mathrm{P}>0.05)$. Thus, it was considered that the movement of smaller for Group 2 and 3 indicated the suddenly changed movement during the hip-lift off phase, although there was no big difference of motion cycle between three groups.

\section{Difference of Knee-Hip Extension Phase}

I was found that Group 3 were much smaller (0.51 \pm 0.06 times) than those for Group $1(\mathrm{P}<0.001)$, although there was no big statistical difference of $(T-T)(P>0.05)$. Those for Group 2 were $0.70 \pm 0.12$, although there was also no big statistical difference of $(T-T)(P>0.05)$. Thus, it was considered that the movement of smaller for Group 2 and 3 indicated the suddenly changed movement during the knee-hip extension phase, although there was no big difference of motion cycle between three groups.

\section{Results of Selection of Activated Muscles}

The active muscles were computed in the three kinds of lifting posture formulated inside Open Sim. The three kinds of lifting posture were reproduced respectively within Open Sim, and an analysis of the static postures related to actuation was conducted in the three lifting postures with the Analyze Tool. Figure 4 shows the results of simulation for two models under the condition of three different postures. It can be seen that the three kinds of postures defined by 3DSSPP have been successfully reproduced similarly. Here, 3DSSPP software predicts static strength requirements for tasks such as lifts, presses, pusses, and pulls. The Center for Ergonomics at the University of Michigan College of Engineering has developed programs for analyzing manual materials-handling tasks. Table 2 shows a list of all activated muscles for the Open Sim model. It can be seen that the type of muscles that are activated differs depending on the type of posture. In this study, we didn't refer to the bilateral difference, therefore, the denominator of muscle is not 96 types but 46 types. As a result, 13/46 type of muscles in "Standing Lift", 13/46 type of muscles in" Stoop Lift" and 16/46 type of muscles in "Squat Lift" was activated. It was identified that a total of activated muscle in the three kinds of lifting posture was 23/46 type of muscles. Based on the below results, the muscles to be activated could be chosen from the defined postures by means of the results of the two simulations. 


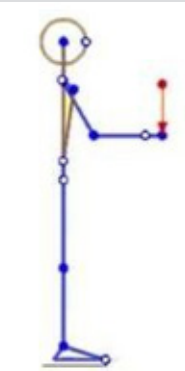

Model of 3DSSPP

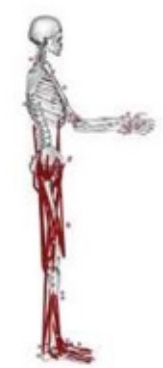

\section{Model of} Opensim

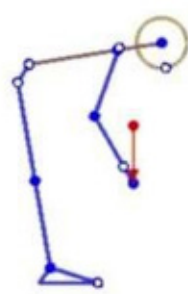

Model of 3DSSPP

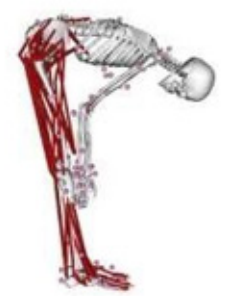

Model of Opensim

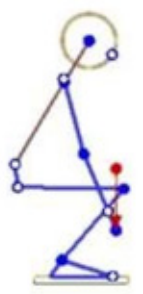

Model of 3DSSPP

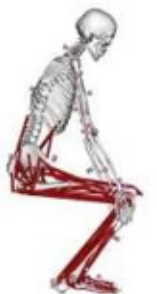

Model of Opensim

(A) Standing lift.

(B) Stoop lift.

(C) Squat lift.

Figure 4: Results of the simulation for two models under the condition of three different postures.

Table 2: The complete list of activated muscles for the OpenSim model.

\begin{tabular}{|c|c|c|c|c|c|}
\hline No. & Open Sim & Muscle name & Standing & Stoop & Squat \\
\hline 1 & Ericson & Erector spine muscle & $\checkmark$ & $\checkmark$ & $\checkmark$ \\
\hline 2 & Bifemlh & Long head of biceps femoris muscle & & $\checkmark$ & $\checkmark$ \\
\hline 3 & Bifemsh & Short head of biceps femoris muscle & $\checkmark$ & $\checkmark$ & $\checkmark$ \\
\hline 4 & vas_int & Vastus intermedius muscle & & & $\checkmark$ \\
\hline 5 & Sar & Sartorius muscle & $\checkmark$ & $\checkmark$ & $\checkmark$ \\
\hline 6 & vas_med & Vastus medialis & & & $\checkmark$ \\
\hline 7 & glut_max1 & Gluteus maximus muscle top & & $\checkmark$ & $\checkmark$ \\
\hline 8 & glut_max2 & Gluteus maximus muscle middle & & $\checkmark$ & $\checkmark$ \\
\hline 9 & glut_max3 & Gluteus maximus muscle bottom & & $\checkmark$ & $\checkmark$ \\
\hline 10 & vas_lat & Vastus lateralis muscle & & & $\checkmark$ \\
\hline 11 & Grac & Gracilis muscle & $\checkmark$ & $\checkmark$ & $\checkmark$ \\
\hline 12 & Semiten & Semitendinosus muscle & $\checkmark$ & $\checkmark$ & $\checkmark$ \\
\hline 13 & add_long & Adductor longus & $\checkmark$ & & \\
\hline 14 & add_brev & Adductor Brevis muscle & $\checkmark$ & $\checkmark$ & $\checkmark$ \\
\hline 15 & add_mag2 & Adductor Magnus muscle middle & & & $\checkmark$ \\
\hline 16 & add_mag3 & Adductor Magnus muscle bottom & & $\checkmark$ & $\checkmark$ \\
\hline 17 & rect_fem & Rectus femoris & $\checkmark$ & & $\checkmark$ \\
\hline 18 & ext_hal & Extensor hallucis longus muscle & $\checkmark$ & $\checkmark$ & \\
\hline 19 & ext_dig & Extensor digitorum brevis muscle & & $\checkmark$ & \\
\hline 20 & Iliacus & Iliopsoas muscle & $\checkmark$ & & \\
\hline 21 & Extobl & Abdominal external oblique muscle & $\checkmark$ & & \\
\hline 22 & Psoas & Psoas major muscle & $\checkmark$ & & \\
\hline 23 & Tfl & Tensor fasciae latae muscle & $\checkmark$ & & \\
\hline
\end{tabular}

\section{Results of Proposed Exercises and Activated Muscles}

Figure 5 shows the results of the proposed static posture through all of the activated muscles. Based on the advice of physical therapists, it was possible to formulate this with exercises in which the workers who were the subjects could move without straining themselves. Table 3 shows the relationship between the activated muscle and static posture. These ten kinds of static posture were rearranged in the order of pelvis $\rightarrow$ hip joint $\rightarrow$ foot $\rightarrow$ ankle, and the order was set as "Forward Bending (FB)," "Back Bending (BB)," "Lateral Bending (LB)," "Horizontal Rotation (HR)," "Vertical Rotation (VR)," Coxae Rotation (CR)," “Coxae Open (CO)," "Lunge (LE)," "Side Lunge (SL)," and "Ankle Rotation (AK)." In addition, the motion time was defined on 3:20 same as radio calisthenics and the flow of a series of exercises was formulated in such a manner that 
it would become these static postures. The three kinds of muscles, muscles in addition to the 23/46 types during lifting. Those muscles separated by a dotted line in Table 3, represent 3/46 types of other are expressed as follows:

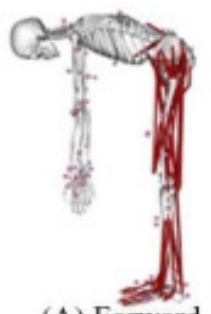

(A) Forward bending $(\mathrm{FB})$.

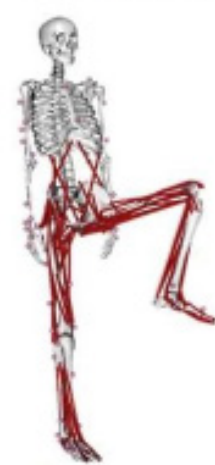

(F) Coxae rotation (CR).

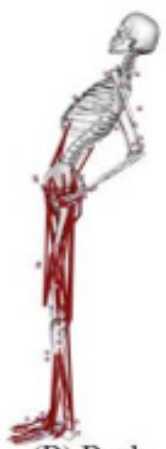

(B) Back bending $(\mathrm{BB})$.

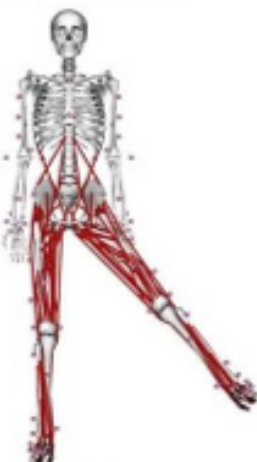

(G) Coxae open (CO).

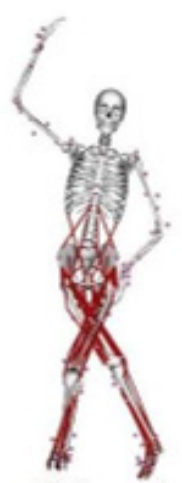

(C) Lateral

bending (LB).

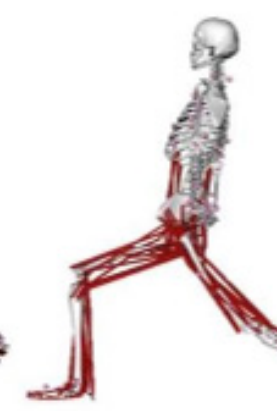

(H) Lunge

(LE).

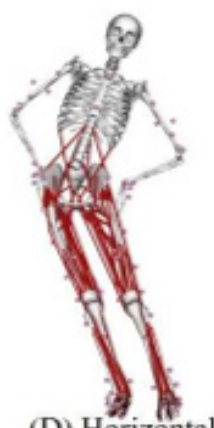

(D) Horizontal rotation (HR).

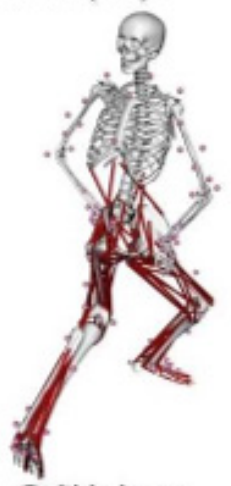

(I) Side lunge

(SL).

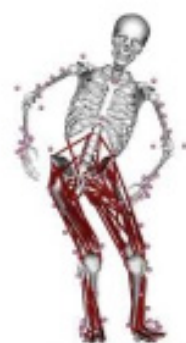

(E) Vertical rotation (VR).

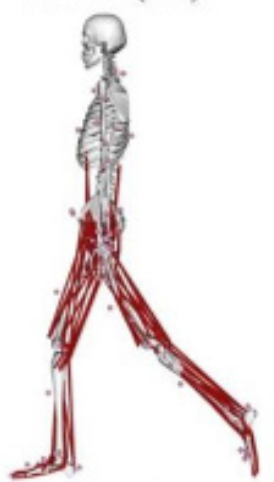

(J) Ankle rotation (AK).

Figure 5: Results of the proposed static posture for all the activated muscles.

Table 3: The relationship between activated muscles and static postures.s

\begin{tabular}{|c|c|c|c|c|c|c|c|c|c|c|c|}
\hline No. & Opensim Muscle & FB & BB & LB & HR & VR & CR & CO & LE & SL & AR \\
\hline 1 & Ercspn & $\checkmark$ & & $\checkmark$ & $\checkmark$ & $\checkmark$ & $\checkmark$ & $\checkmark$ & $\checkmark$ & $\checkmark$ & $\checkmark$ \\
\hline 2 & Bifemlh & & & & $\checkmark$ & & $\checkmark$ & $\checkmark$ & $\checkmark$ & $\checkmark$ & $\checkmark$ \\
\hline 3 & Bifemsh & $\checkmark$ & $\checkmark$ & $\checkmark$ & $\checkmark$ & $\checkmark$ & $\checkmark$ & $\checkmark$ & $\checkmark$ & \\
\hline 4 & vas_int & & & & & $\checkmark$ & $\checkmark$ & & & \\
\hline 5 & Sar & $\checkmark$ & $\checkmark$ & $\checkmark$ & $\checkmark$ & $\checkmark$ & $\checkmark$ & $\checkmark$ & $\checkmark$ & $\checkmark$ & $\checkmark$ \\
\hline 6 & vas_med & & & & & $\checkmark$ & $\checkmark$ & & & \\
\hline 7 & glut_max1 & & & $\checkmark$ & & & $\checkmark$ & & $\checkmark$ & \\
\hline 8 & glut_max2 & & & $\checkmark$ & & & $\checkmark$ & & $\checkmark$ & $\checkmark$ & $\checkmark$ \\
\hline 9 & glut_max3 & & & $\checkmark$ & $\checkmark$ & $\checkmark$ & $\checkmark$ & $\checkmark$ & $\checkmark$ & $\checkmark$ & $\checkmark$ \\
\hline 10 & vas_lat & & & & & $\checkmark$ & $\checkmark$ & & & \\
\hline 11 & grac & $\checkmark$ & $\checkmark$ & $\checkmark$ & $\checkmark$ & $\checkmark$ & $\checkmark$ & $\checkmark$ & $\checkmark$ & $\checkmark$ & $\checkmark$ \\
\hline 12 & semiten & $\checkmark$ & $\checkmark$ & $\checkmark$ & $\checkmark$ & $\checkmark$ & $\checkmark$ & $\checkmark$ & $\checkmark$ & $\checkmark$ & $\checkmark$ \\
\hline 13 & add_long & $\checkmark$ & $\checkmark$ & & $\checkmark$ & $\checkmark$ & $\checkmark$ & $\checkmark$ & $\checkmark$ & $\checkmark$ & $\checkmark$ \\
\hline 14 & add_brev & $\checkmark$ & $\checkmark$ & $\checkmark$ & & $\checkmark$ & $\checkmark$ & $\checkmark$ & $\checkmark$ & $\checkmark$ & $\checkmark$ \\
\hline 15 & add_mag2 & & & & & & $\checkmark$ & $\checkmark$ & $\checkmark$ & $\checkmark$ & $\checkmark$ \\
\hline 16 & add_mag3 & & & $\checkmark$ & $\checkmark$ & $\checkmark$ & $\checkmark$ & $\checkmark$ & $\checkmark$ & $\checkmark$ & $\checkmark$ \\
\hline 17 & rect_fem & $\checkmark$ & $\checkmark$ & $\checkmark$ & $\checkmark$ & $\checkmark$ & $\checkmark$ & $\checkmark$ & $\checkmark$ & $\checkmark$ & $\checkmark$ \\
\hline 18 & ext_hal & $\checkmark$ & $\checkmark$ & $\checkmark$ & $\checkmark$ & & $\checkmark$ & $\checkmark$ & $\checkmark$ & $\checkmark$ \\
\hline
\end{tabular}




\begin{tabular}{|c|c|c|c|c|c|c|c|c|c|c|c|}
\hline 19 & ext_dig & & & & $\checkmark$ & & & & & & $\checkmark$ \\
\hline 20 & iliacus & $\checkmark$ & $\checkmark$ & $\checkmark$ & $\checkmark$ & $\checkmark$ & $\checkmark$ & $\checkmark$ & $\checkmark$ & $\checkmark$ & $\checkmark$ \\
\hline 21 & extobl & & $\checkmark$ & $\checkmark$ & $\checkmark$ & & $\checkmark$ & $\checkmark$ & $\checkmark$ & $\checkmark$ & $\checkmark$ \\
\hline 22 & psoas & $\checkmark$ & $\checkmark$ & $\checkmark$ & $\checkmark$ & $\checkmark$ & $\checkmark$ & $\checkmark$ & $\checkmark$ & $\checkmark$ & $\checkmark$ \\
\hline 23 & $\mathrm{tfl}$ & $\checkmark$ & $\checkmark$ & $\checkmark$ & $\checkmark$ & $\checkmark$ & $\checkmark$ & $\checkmark$ & $\checkmark$ & & $\checkmark$ \\
\hline 1 & add_mag1 & & & $\checkmark$ & & & $\checkmark$ & $\checkmark$ & & $\checkmark$ & \\
\hline 2 & tib_ant & & & & & & & & & & $\checkmark$ \\
\hline 3 & intobl & & $\checkmark$ & $\checkmark$ & $\checkmark$ & & $\checkmark$ & & $\checkmark$ & $\checkmark$ & \\
\hline
\end{tabular}

I. *add_mag1 = Adductor Magnus muscle top,

II. *tib_ant $=$ Tibialis anterior muscle,

III. *intobl = Abdominal internal oblique muscle.

Based on the below results, it can be confirmed that the muscles selected in the simulation are activated by the proposed warm-up exercises.

\section{Results of The Evaluation of Exercise Effects}

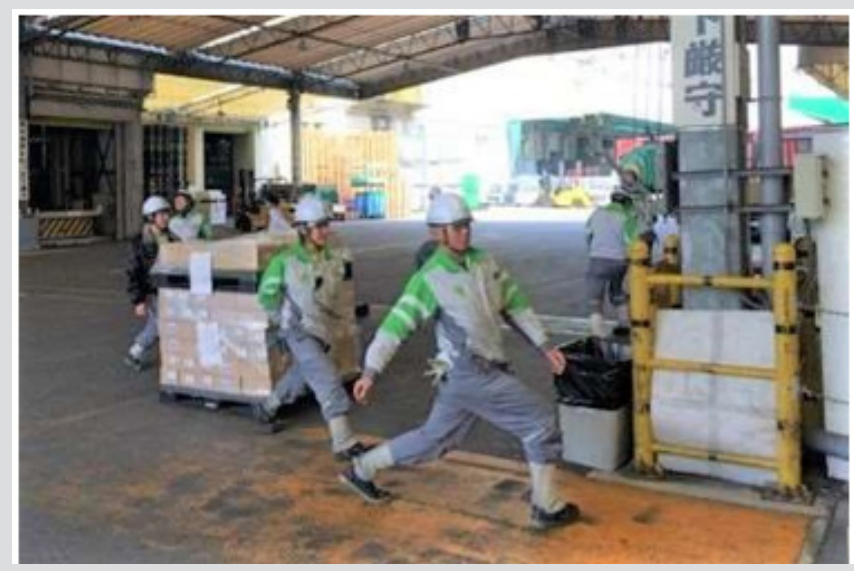

Figure 6: Circumstances of conducting exercise for the clinical test.
Figure 6 shows the circumstances of conducting the exercise. In this trial, the number of cases was small at six (Age: $38 \pm 10.02$, Weight: $68.3 \pm 7.12$, Hight: $170.3 \pm 4.63$ ) in Group 3 , so it seemed that it was not necessary to perform the statistical analysis. The changes and medians of the respective subjects were evaluated. Figure 7 shows one of the results of the effect of warm-up exercise on evaluating items. Figure 7 (A) shows the results for the VAS. These figures decreased for all 6 subjects, and along with this, the median decreased from 39.5 to 16 . As for the results for the evaluation items related to QOL. The median of ODI was a tendency to decrease, from 15 to 12 . Figure 7 (B) shows the results for the RDQ. The figures decreased for all 6 subjects, and along with this, the median decreased from 1 to 0 . The median of QOL SF-36 v2 PCS was almost no change, from 54.1 to 54.4 , QOL SF-36 v2 MCS was almost no change, from 49.7 to 52.0 and QOL SF-36 v2 RCS was almost no change in the median, from 49.3 to 48.9. As for the results of items related to physical measurements. Figure 7 (C) shows the results of FFD. The figures rose for all 6 subjects, and along with this, the median rose from -5.5 to 2.8. The median of MM was no change at all. The median of the external rotation angle of the right foot was a tendency to rise, from 29 to 36 and the external rotation angle of the left foot was a tendency to rise, from 33 to 39. The median of the abduction angle of the right foot was a tendency to rise, from 52 to 60 and the abduction angle of the left foot was a tendency to rise, from 51 to 62 .

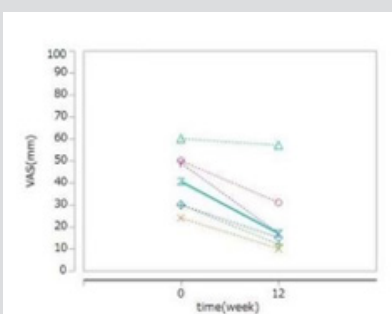

(A) VAS(mm).

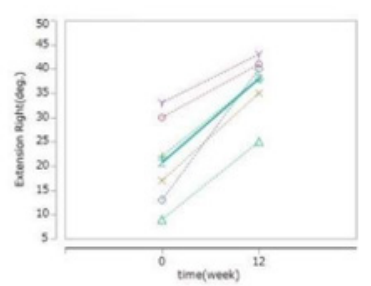

(D) Extention Right(deg.).

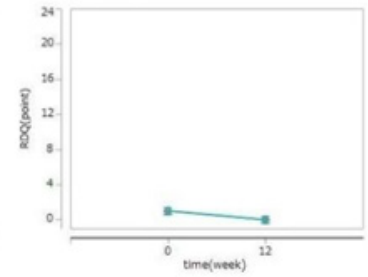

(B) RDQ(point),

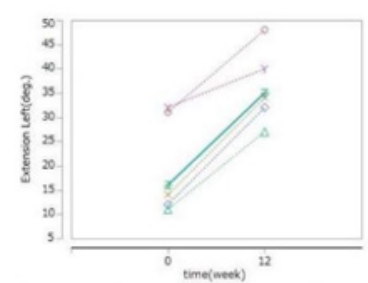

(E) Extention Left(deg.).

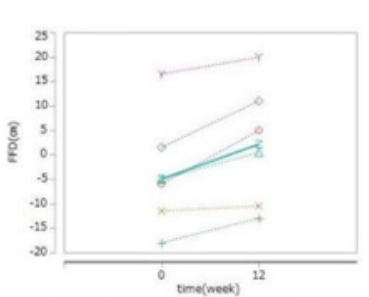

(C) $\mathrm{FFD}(\mathrm{cm})$.

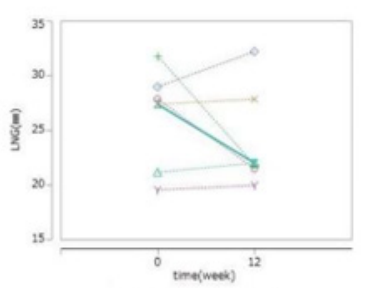

(F) $\mathrm{LNG}(\mathrm{mm})$.

Figure 5: Results of the proposed static posture for all the activated muscles. 
The median of the flexion angle of the right foot was a tendency to rise, from 99 to 108 and the flexion angle of the left foot was almost no change in the median, from 103 to 104. Figure 7 (D) shows the results for the extension angle of the right foot. These figures rose for all 6 subjects, and along with this, the median also rose greatly, from 20 to 39 . Figure 7 (E) shows the results for the extension angle of the left foot. These figures rose for all 6 subjects, and along with this. The median also rose greatly, from 15 to 35. The median of the internal rotation angle of the right foot rose greatly, from 24 to 45 and the internal rotation angle of the left foot also rose from 27 to 49 . The median of the adduction angle of the right foot rose from 29 to 31 and the adduction angle of the left foot rose greatly from 28 to 38 . Figure 7 (F) shows the results for LNG of CoP. The figures decreased for only 3 subjects, and the median decreased slightly, from 27.6 to 21.9. The median of SV of CoP decreased slightly, from 9.2 to 7.3 and EA of CoP was a tendency to decrease, from 190.0 to 181.3 .

\section{Discussion}

\section{Feasibility of The Proposed Protocol for Warm-Up Exercise}

The 23/46 types of muscles that are activated during the lifting of heavy objects were calculated with heavy object lifting postures as well as musculoskeletal simulations. In addition, 10 kinds of static postures for thoroughly activating these active muscles were formulated in concert with a physical therapist, and the protocol of the method for formulating a series of exercises was indicated. By means of that protocol, exercises that thoroughly moved the muscles that are activated during heavy object lifting were formulated. It is believed that a large number of muscles of the human body are activated in complex ways during the lifting of heavy objects. It is difficult to evaluate these by actual measurements. As a result, the active muscles that serve as the axis were calculated with musculoskeletal simulation software. It was common to select and formulate exercises by the rule of thumb, but by focusing attention on the movement of the muscles in the musculoskeletal, this suggests the possibility of examining the differences between exercises and the type of exercise.

\section{Limitation of Stretching Exercise at The Logistic Work-} place

In the intervention trial phase, the 12 -week intervention was implemented, and verification of the effects was confirmed. It was considered that the improvement of these evaluating indices had potential preventive effects. Through the results, the stretching exercise prevented logistics workers from the work-related MSDs. Pain from MSDs was improved due to the improvement of VAS. The improvement of the RDQ median indicated the ability of the low back. Through the results of physical examination, it was found that the flexibility enhanced due to the improvement of FFD. Moreover, the ROM of the lower limb joints was observed particularly in the extended angle. The proposed stretching exercise was effective in improving the ROM of these joints. Since it was believed that the flexibility and the ROM of lower limb joints are a cause of the onset of MSDs pain, there was a possibility that the improvement of these causes can produce an expectation of preventive effects on workrelated disorders. However, the limitation of this experiment is that expected activated 26 kinds of muscles are confirmed by the simulation without any measurement and the muscle strength is not affected by the stretching exercise through the results of measured muscle mass.

\section{Conclusion}

The proposed stretching exercise as one kind of rehabilitation was effective through the results of the clinical test at the workplace, which was formulated by the simulation of Open Sim. A protocol for formulating warm-up exercises for the work-related low back disorders of logistics workers with musculoskeletal simulations was proposed. In addition, a clinical trial was implemented to evaluate the effects, and it suggested the potential for ameliorative effects on work-related low back disorders. Through both qualitative and quantitative evaluation, it was found that the proposed warm-up exercise enabled workers to improve the muscle flexibility as the quantitative evaluation, then the results of qualitative evaluation including the quality of life, visual analysis scale, and so on were connected to the improvement. In the future, we will continue to increase the number of cases and conduct RCT trials and to verify the effects of trials with a higher standard of evidence.

\section{Acknowledgment}

I would like to thank Katsutoshi Kuwabara and Takeshi Terada to cooperate in conducting a clinical trial. This study was a part of a joint research with KONOIKE Transport CO., LTD.

\section{Conflict of Interest}

The authors declare that there are no conflicts of interest.

\section{References}

1. E Schneider, X Irastorza (2010) OSH in figures: Work-related musculoskeletal disorders in the EU-Facts and figures. European Agency for Safety and Health at Work, European risk observatory report.

2. H Suzuki, T Kanchiku, Y Imajo, Y Yoshida, N Nishida, et al. (2016) Diagnosis and Characters of Non-Specific Low Back Pain in Japan: The Yamaguchi Low Back Pain Study. PLoS One 11(8): e0160454.

3. H Jeong, Y Ohno (2017) Symmetric lifting posture recognition of skilled experts with linear discriminant analysis by center-of-pressure velocity. Intelligent Service Robotics 10(4): 323-332.

4. H Jeong, K Yamada, M Kido, S Okada, T Nomura, et al. (2016) Analysis of difference in center-ofpressure positions between experts and novices during asymmetric lifting. IEEE Journal of Translational Engineering in Health and Medicine 4: 2100311.

5. J Mayer, V Mooney, S Dagenais (2008) Evidence-informed management of chronic low back pain with lumbar extensor strengthening exercises. Spine J 8(1): 96-113.

6. O Airaksinen, J I Brox, C Cedraschi, J Hildebrandt, J Klaber-Moffett, et al. (2006) COST B13 Working Group on Guidelines for Chronic Low Back Pain. European guidelines for the management of chronic nonspecific low back pain, Chapter 4 Eur Spine J 15(2): 192-300. 
7. (2018) Konoike Transport Company.

8. Y Ouchi, H Okada, E Yoshikawa, S Nobezawa, M Futatsubashi, et al (1999) Brain activation during maintenance of standing postures in humans. Brain 122 (2): 329-338.

9. (2018) 3DSSPP.

10. (2018) Open Sim

11. P Magnusson, P Renström (2006) The European College of Sports Sciences Position statement: The role of stretching exercises in sports European Journal of Sport Science 6(2): 87-91.

12. Lippincott Williams, Wilkins (2017) American College of Sports Medicine. ACSM's Guidelines for Exercise Testing and Prescription.

13. M Roland, J Fairbank (2000) The Roland-Morris Disability Questionnaire and the Oswestry Disability Questionnaire. Spine (Phila Pa 1976) 25(24): 3115-3124.

14. M Roland, R Morris (1983) A study of the natural history of back pain Part I: development of a reliable and sensitive measure of disability in low-back pain. Spine (Phila Pa 1976) 8(2): 141-144.

\section{ISSN: 2574-1241}

DOI: 10.26717.BJSTR.2019.14.002542

Hieyong Jeong. Biomed J Sci \& Tech Res

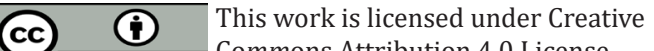

Submission Link: https://biomedres.us/submit-manuscript.php
15. M C Battié, S J Bigos, L D Fisher, D M Spengler, T H Hansson, et al. (1990) The role of spinal flexibility in back pain complaints within industry. A prospective study. Spine (Phila Pa 1976) 15(8): 768-773.

16. LR Van Dillen, N J Bloom, SP Gombatto, TM Susco (2008) Hip rotation range of motion in people with and without low back pain who participate in rotation-related sports. Phys Ther Sport 9(2): 72-81.

17. EP Takala, E Viikari-Juntura (2000) Do functional tests predict low back pain? Spine (Phila Pa 1976) 25(16): 2126-2132.

18. R A Clark, A L Bryant, Y Pua, P McCrory, K Bennell, et al. (2010) Validity and reliability of the Nintendo Wii Balance Board for assessment of standing balance. Gait Posture 31(3): 307-310.

19. D S Park, G Lee (2014) Validity and reliability of balance assessment software using the Nintendo Wii balance board: Usability and validation. J Neuro Eng Rehabil 11: 1-8.

20. M Vukobratovic, B Borovac (2004) Zero-moment point - Thirty-five years of its life. Int. J. Humanoid Robot 1(1): 157-173.

$\begin{array}{ll}\text { BIOMEDICAL } & \text { Assets of Publishing with us } \\ \text { RESEARCHES } & \text { - Global archiving of articles } \\ \text { - Immediate, unrestricted online access }\end{array}$

